Pacific Journal of Mathematics

A GREEN'S FUNCTION APPROACH TO PERTURBATIONS OF 


\section{A GREEN'S FUNCTION APPROACH TO PERTURBATIONS OF PERIODIC SOLUTIONS}

Carl Kallina

Consider the nonlinear differential system

$$
\dot{y}=A(t) \cdot y+\varepsilon q(t, y, \varepsilon)
$$

where $y, q$ are column $n$-vectors, $q$ is continuous in $(t, y, \varepsilon)$ and has continuous second partial derivatives with respect to $y, \varepsilon$ for all values of $t, 0 \leqq y \leqq R$ for some $R>0$ and $0 \leqq \varepsilon \leqq \varepsilon_{0}$ for some $\varepsilon_{0}>0$. Further assume $A(t)$ is an $n \times n$ matrix such that $A \in C^{1}$, and both $A$ and $q$ are periodic in $t$ with period $T$. Associated with system (1.1) are the general homogeneous and nonhomogeneous equations

$$
\begin{gathered}
\dot{y}=A(t) \cdot y \\
\dot{y}=A(t) \cdot y+f(t)
\end{gathered}
$$

where $f(t)$ is an arbitrary $n$-vector function periodic in $t$ of period $T$. In this paper we consider the classical problem of proving the existence of $T$-periodic solutions $y=y(t)$ of $(1.1)$ when the homogeneous system $(1.2)$ has nontrivial $T$-periodic solutions.

In his book "Oscillations in Nonlinear Systems" [5], J. K. Hale treats this problem in Chapter 11 as an extension of the general method developed in Chapter 6 for the case when $A(t)$ is a constant matrix $A$. Among other treatments of this problem is the work of D. C. Lewis [6] utilizing a generalized Green's matrix. It is the aim of the present paper to incorporate certain ideas from [6] into the general method employed in [5] in Chapter 6. We feel that the approach presented here brings into sharper focus the generality of the methods employed in [5] and also has certain computational advantages which will be mentioned in the body of the paper. The present paper has many points of contact with a recent paper of S. Bancroft, J. K. Hale, and D. Sweet [2]. See also [1], [7] and [8].

2. We must first consider the general nonhomogeneous equation (1.3). We will state a necessary and sufficient condition for the existence of $T$-periodic solutions of (1.3) when (1.2) has nontrivial $T$ periodic solutions. Following [6] we then construct a generalized Green's function in order to find an explicit periodic solution of (1.3).

Let $S=\{f: f(t)=f(t+T) ; f \in C$; and $f(t)$ is a real valued $n$-vector for all $t$. Then $S$ is a Banach space if we define the norm $V(f)$ to 
be $V(f)=\sup \{\|f(t)\| ; 0 \leqq t \leqq T\}$. Further let $X(t)$ be any real fundamental matrix solution of (1.2), and let $n-k$ be the rank of the $n \times n$ matrix $L=X(0)-X(T)$. Then there exist $k$ linearly independent column vectors $b_{1}, b_{2}, \cdots, b_{k}$ such that $L \cdot b_{i}=0$ for $i=1,2, \cdots, k$. Let $B$ denote the $n \times k$ matrix $\left(b_{1}, b_{2}, \cdots, b_{k}\right)$. Then the columns $\varphi_{1}(t), \varphi_{2}(t), \cdots, \varphi_{k}(t)$ of the matrix $\Phi(t)$ defined by $\Phi(t)=X(t) \cdot B$ form a set of $k$ linearly independent solutions of (1.2) which are $T$-periodic. By assumption $k>0$, and of course the columns of $\Phi(t)$ span the space $S_{1}$ of all $T$-periodic solutions of (1.2). Explicitly we have

$$
S_{1}=\left\{f \in S: f(t)=\Phi(t) \cdot a=\sum a_{i} \cdot \varphi_{i}(t) \text { for all } a=\left(a_{1}, a_{2}, \cdots, a_{k}\right) \in R^{k}\right\} \text {. }
$$

Let $A^{\prime}$ denote the transpose of an $r \times s$ matrix $A$. Let $N(A)$ and $R(A)$ denote the nullity and range of $A$, respectively. Consider the $n \times n$ matrix $L^{\prime}=X^{\prime}(0)-X^{\prime}(T)$. Since $\operatorname{dim} N\left(L^{\prime}\right)=\operatorname{dim} N(L)$, we can find $k$ linearly independent column vectors $u_{1}, u_{2}, \cdots, u_{k}$ such that $L^{\prime} \cdot u_{i}=u_{i}^{\prime} \cdot L=0$ for $i=1,2, \cdots, k$. Let $U$ denote the $k \times n$ matrix with rows $u_{1}^{\prime}, u_{2}^{\prime}, \cdots, u_{k}^{\prime}$. We define an $n \times k$ matrix $\Psi(t)$ to be

$$
\Psi(t)=\left[U \cdot X(T) \cdot X^{-1}(t)\right]^{\prime} .
$$

Then $\Psi^{\prime}(t)=U \cdot X(T) \cdot X^{-1}(t)$. Clearly, $\Psi(t)$ has rank $k$ and each of its columns is a solution of the differential equation

$$
\dot{Z}=-A^{\prime}(t) \cdot Z
$$

which is adjoint to (1.2).

Let $\psi_{1}(t), \psi_{2}(t), \cdots, \psi_{k}(t)$ denote the $k$ linearly independent columns of $\Psi(t)$. These functions span the space $S_{2}$ of all $T$-periodic solutions of the adjoint equation (2.1). In fact, since $\Psi(0)=\Psi(T)$ and $\Psi(t)$ is a matrix solution of (2.1) we see that $\Psi(s)=\Psi(s+T)$ for $0 \leqq s \leqq T$. If (2.1) had $l$ linearly independent periodic solutions with $l>k$ then the original system (1.2), which is adjoint to (2.1), would also have at least $l$ linearly independent $T$-periodic solutions, contrary to assumption. Explicitly, we have

$$
S_{2}=\left\{f \in S: f(t)=\Psi(t) \cdot a=\sum a_{i} \cdot \psi_{i}(t) \text { for all } a=\left(a_{1}, a_{2}, \cdots, a_{k}\right) \in R^{k}\right\} \text {. }
$$

It is easy to see that $S$ becomes a real Euclidean space when an inner product $(f, g)$ is defined for $f, g \in S$ by

$$
(f, g)=\frac{1}{T} \cdot \int_{0}^{T}\langle f(t), g(t)\rangle d t=\sum_{i=1}^{n} \frac{1}{T} \cdot \int_{0}^{T} f_{i}(t) \cdot g_{i}(t) d t
$$

where $\langle a, b\rangle=\sum_{i=1}^{n} a_{i} \cdot b_{i}$ denotes the usual inner product for $a, b \in R^{n}$. Without loss of generality we may assume that both $\left\{\varphi_{i}(t)\right\}$ and $\left\{\psi_{i}(t)\right\}$ are orthonormal bases for their respective subspaces $S_{1}$ and $S_{2}$; i.e., 
$\left(\varphi_{i}, \varphi_{j}\right)=\left(\psi_{i}, \psi_{j}\right)=\delta i j$ for $1 \leqq i, j \leqq k$.

By $P$ and $Q$ we denote the orthogonal projections of $S$ onto $S_{1}$ and $S_{2}$, respectively. Explicitly we have

$$
P: S \rightarrow S_{1}, \quad P f(t)=\sum_{i=1}^{n}\left(f, \varphi_{i}\right) \cdot \varphi_{i}(t)
$$

$$
Q: S \rightarrow S_{2}, \quad Q f(t)=\sum_{i=1}^{n}\left(f, \psi_{i}\right) \cdot \psi_{i}(t)
$$

For convenience we summarize certain well-known general properties of projections in Euclidean space.

(i) $f \in S_{1} \Rightarrow P f=f$ and $f \in S_{2} \Rightarrow Q f=f$.

(ii) $P^{2} f=P f$ and $Q^{2} f=Q f$ for all $f \in S$.

(iii) $P f=0 \Longleftrightarrow\left(f, \varphi_{i}\right)=0$ for $i=1,2, \cdots k$.

(iv) $Q f=0 \Longleftrightarrow\left(f, \psi_{i}\right)=0$ for $i=1,2, \cdots k$.

Let us consider the condition $Q f=0$. By (iv) we have $Q f=0 \Longleftrightarrow$

$$
0=\left(f, \psi_{i}\right)=\left(\psi_{i}, f\right)=\frac{1}{T} \int_{0}^{T}\left\langle\psi_{i}(t), f(t)\right\rangle d t
$$

for $i=1,2, \cdots, k$. In matrix notation these $k$ equations can be written as

$$
0=\int_{0}^{T} \Psi^{\prime}(t) \cdot f(t) d t=\int_{0}^{T} U \cdot X(T) \cdot X^{-1}(t) \cdot f(t) d t .
$$

Again using matrix notation we note explicitly that for $f \in S$ we have

$$
Q f(t)=\Psi(t) \cdot \frac{1}{T} \cdot \int_{0}^{T} \Psi^{\prime}(s) \cdot f(s) d s .
$$

Lemma 1. The differential system (1.3) with $f \in S$ has a $T$ periodic solution if and only if $Q f=0$.

Proof. It is well known that the unique solution $y(t)$ of (1.3) satisfying $y(0)=y_{0}$ is given by

$$
y(t)=X(t) \cdot X^{-1}(0) \cdot y_{0}+\int_{0}^{t} X(t) \cdot X^{-1}(s) \cdot f(s) d s .
$$

Let $\beta=X^{-1}(0) \cdot y_{0}$. The solution $y(t)$ is $T$-periodic if and only if $y(0)=$ $y(T)$, and from (2.7) this is equivalent to the statement that the matrix equation

$$
L \cdot \beta=\int_{0}^{T} X(T) \cdot X^{-1}(s) \cdot f(s) d s
$$

can be solved for $\beta$. Suppose first that such a vector $\beta$ has been 
found. The matrix $U$ was defined in such a way that $U \cdot L=0$. Multiplying (2.8) by $U$ and making use of (2.5) we see that $Q f=0$.

Conversely suppose $Q f=0$. It follows from (2.5) that for each column $u_{i}$ of the matrix $U^{\prime}$ we have

$$
\left\langle u_{i}, \int_{0}^{T} X(T) \cdot X^{-1}(s) \cdot f(s) d s\right\rangle=0 .
$$

Thus for each vector $x \in N\left(L^{\prime}\right)$ we have

$$
\left\langle x, \int_{0}^{T} X(T) \cdot X^{-1}(s) \cdot f(s) d s\right\rangle=0 .
$$

From the compatibility theorem of linear algebra this guarantees the existence of some solution of (2.8).

For an arbitrary linear equation $L \cdot \beta=\gamma$, it is known that one can find a nonsingular $n \times n$ matrix $M$, called a pseudoinverse of $L$, such that $L \cdot M \cdot L=L$ and for each $\gamma \in R(L)$ we have $L \cdot(M \cdot \gamma)=\gamma$. Pseudo inverses are constructed from the given matrix $L$ by means of elementary row operations (see [3], [4]). The following corollary is an immediate consequence of this observation.

CoROLlary. If $f \in S$ and $Q f=0$, then (2.8) has a solution of the form $\beta=\int_{0}^{T} P(s) \cdot f(s) d s$, where $P(s)=M \cdot X(T) \cdot X^{-1}(s)$ and $M$ is a nonsingular pseudo inverse of $L$.

Lemma 2. Consider (1.3) and suppose $f \in S$ and $Q f=0$. Then there exists an $n \times n$ matrix $G(t, s)$ which is independent of the function $f$ and continuous in $t$ and $s$ except at $t=s$ where it has a finite jump, and such that

( i ) The function $y_{0}(t)=\int_{0}^{T} G(t, s) \cdot f(s) d s$ is a T-periodic solution of (1.3) and moreover is the only T-periodic solution satisfying $P y_{0}=0$.

(ii) The columns of $\Psi(s)$ are orthogonal to the rows of $G(t, s)$ for each $t$; i.e.,

$$
\int_{0}^{T} G(t, s)^{0} \cdot \Psi(s) d s=0
$$

Proof. Since $Q f=0,(2.8)$ has a solution $\beta$ of the form indicated in the preceding corollary. Substituting $\beta$ into (2.7) we get the equation

$$
y(t)=\int_{0}^{t} X(t) \cdot\left[P(s)+X^{-1}(s)\right] \cdot f(s) d s+\int_{t}^{T} X(t) \cdot P(s) \cdot f(s) d s,
$$

or 


$$
\begin{array}{rlrl}
y(t)=\int_{0}^{T} & K(t, s) \cdot f(s) d s & \\
K(t, s) & =X(t) \cdot\left[P(s)+X^{-1}(s)\right] & & 0 \leqq s \leqq t \\
& =X(t) \cdot P(s) & & t \leqq s \leqq T
\end{array}
$$

for the unique $T$-periodic solution of (1.3) satisfying $y(0)=y_{0}$. Thus any $T$-periodic solution of (1.3) will be in the form

$$
y(t)=\Phi(t) \cdot c+\int_{0}^{T} K(t, s) \cdot f(s) d s
$$

for a suitable choice of $c \in R^{k}$.

It is easy to see that at most one $T$-periodic solution $y_{0}$ of (1.3) can satisfy $P y_{0}=0$. For if $y^{*} \in S$ is another solution with $P y^{*}=0$, then $y=y_{0}-y^{*} \in S$ and solves (1.2). Thus $y \in S_{1}$ and so $P y=y$. On the other hand we have $P y=P y_{0}-P y^{*}=0$, which implies $y_{0}=y^{*}$.

To verify (i), we first observe that the condition $P y=0$ is equivalent to the equation

$$
\int_{0}^{T} \Phi^{\prime}(t) \cdot y(t) d t=0
$$

We want to find $c \in R^{k}$ such that both (2.11) and (2.12) are satisfied. For this it is necessary and sufficient that

$$
0=\frac{1}{T} \int_{0}^{T} \Phi^{\prime}(t) \cdot \Phi(t) \cdot c d t+\frac{1}{T} \int_{0}^{T} \Phi^{\prime}(t) \cdot\left[\int_{0}^{T} K(t, s) \cdot f(s) d s\right] d t .
$$

Since $\left\{\varphi_{i}(t)\right\}$ forms an orthonormal basis,

$$
\frac{1}{T} \int_{0}^{T} \Phi^{\prime}(t) \cdot \Phi(t) d t=I(k \times k)
$$

and from $\Phi(t)=X(t) \cdot B$ we find

$$
c=-\frac{1}{T} \int_{0}^{T} B^{\prime} \cdot\left[\int_{0}^{T} X^{\prime}(T) \cdot K(t, s) d t\right] \cdot f(s) d s .
$$

Thus the vector $c$ is completely determined by an equation of the form

$$
c=\int_{0}^{T} \mathscr{L}(s) \cdot f(s) d s
$$

where $\mathscr{L}(s)$ for a given $K(t, s)$ is a continuous $k \times n$ matrix function of $s$, independent of $f$, given explicitly by (2.13). Substituting (2.14) into (2.11) and introducing the function

$$
G^{*}(t, s)=\Phi(t) \cdot \mathscr{L}(s)+K(t, s)
$$

we find the $T$-periodic solution of (1.3) satisfying $P y_{0}=0$ to be 


$$
y_{0}(t)=\int_{0}^{T} G^{*}(t, s) \cdot f(s) d s .
$$

From (2.5), however, we see that for any $n \times k$ matrix function $R(t)$ the solution $y_{0}(t)$ in $(2.16)$ can also be written as

$$
y_{0}(t)=\int_{0}^{T}\left[G^{*}(t, s)-R(t) \cdot \Psi^{\prime}(s)\right] \cdot f(s) d s .
$$

Choosing in particular the matrix

$$
R(t)=\frac{1}{T} \int_{0}^{T} G^{*}(t, s) \cdot \Psi(s) d s
$$

and recalling that $\left\{\psi_{i}(t)\right\}$ forms an orthonormal basis, we see that (2.9) is satisfied for

$$
G(t, s)=G^{*}(t, s)-R(t) \cdot \Psi^{\prime}(s) .
$$

Finally, it is clear that $G(t, s)$ has the specified smoothness properties.

Corollary. Let $f \in S$ and $Q f=0$. The unique solution $y_{0}(t)$ of (1.3) with $P y_{0}=0$ satisfies the inequality

$$
V\left(y_{0}\right) \leqq K \cdot V(f)
$$

for $K=T \cdot \sup \{\|G(t, s)\|: 0 \leqq s, t \leqq T\}$.

Lemma 3. The matrix $G(t, s)$ given above is uniquely determined by its properties specified in the statement of Lemma 2.

Proof. Let $g(s)$ be any element of $S$. Set $f(s)=g(s)-Q g(s)$. Then $Q f=Q g-Q^{2} g=0$ and so we may apply Lemma 1. From (2.6) and (2.9), however, we find that

$$
\int_{0}^{T} G(t, s) \cdot f(s) d s=\int_{0}^{T} G(t, s) \cdot g(s) d s
$$

and so the unique solution $y_{0}(t)$ satisfying $P y_{0}=0$ is given by

$$
y_{0}(t)=\int_{0}^{T} G(t, s) \cdot g(s) d s \text {. }
$$

If $G_{1}(t, s)$ were a second matrix with the properties given in Lemma 2, then the same argument shows that

$$
y_{0}(t)=\int_{0}^{T} G_{1}(t, s) \cdot g(s) d s
$$

as well. From 


$$
\int_{0}^{T}\left[G(t, s)-G_{1}(t, s)\right] \cdot g(s) d s=0
$$

it follows immediately that $G_{1}(t, s)=G(t, s)$.

REMARKs. The subspaces $S_{1}$ and $S_{2}$ were introduced by Hale in [5]. The proofs of Lemma 1,2 and 3 are modeled on the treatment in [6]. A discussion of these ideas employing the Moore-Penrose notion of generalized inverse can be found in [7]. It turns out that the estimate (2.18) is the essential information required for the iteration scheme which we construct in the next section. We note, however, that the existence of the function $G(t, s)$ has been established by constructive methods which in principle make it possible to write out explicitly every $T$-periodic solution of (1.3) for arbitrary $f \in S$.

3. In this section we indicate how the above analysis can be applied to the question of the existence of periodic solutions of (1.1) for $\varepsilon \neq 0$. Our treatment is a modification of the analysis in Chapters 6 and 11 of [5], and the reader is referred there for details and further results.

Let $a^{*}(t)$ be any $T$-periodic solution of (1.2). For some $a \in R^{k}$ we have $P a^{*}(t)=a^{*}(t)=\Phi(t) \cdot a$. For given constants $b, d$ with $0<b<d<R$ and $a^{*}(t) \in S_{1}$ we define $S_{0} \subset S$ as

$$
S_{0}=\left\{f \in S: P f(t)=a^{*}(t) ; V(f) \leqq d\right\} .
$$

TheOREM 1. Given a constant $b, 0<b \leqq R$, there exists an $\varepsilon_{1}>0$ such that corresponding to each $k$-vector function $a^{*}(t) \in S_{1}$ with $a^{*}(t)=\Phi(t) \cdot a$ and $V\left(a^{*}\right) \leqq b$ and to each $\varepsilon$ with $|\varepsilon| \leqq \varepsilon_{1}$, there is $a$ unique function $y^{*}(t)=y^{*}(t, a, \varepsilon) \in S_{0}$ which has a continuous first derivative with respect to $t$, satisfies $y^{*}(t, a, 0)=a^{*}(t)$ and

$$
\dot{y}^{*}=A(t) \cdot y^{*}+\varepsilon \cdot\left[q\left(t, y^{*}, \varepsilon\right)-Q q\left(t, y^{*}, \varepsilon\right)\right] \text {. }
$$

Finally, the function $y^{*}(t)$ may be obtained by the iteration procedure in (3.4) below.

Proof. Since the function $G(t, s)$ constructed in Lemma 2 is independent of $f \in S$, it makes sense to define an operator $\mathscr{F}$ for $y=$ $y(t, a, \varepsilon)$ in $S_{0}$ as

$$
w=\mathscr{F} y=a^{*}(t)+\varepsilon \cdot \int_{0}^{T} G(t, s) \cdot q(s, y(s, a, \varepsilon), \varepsilon) d s .
$$

As in the proof of Lemma 3 we see that the function $Z(t)$ defined by

$$
Z(t)=\varepsilon \cdot \int_{0}^{T} G(t, s) \cdot q(s, y(s, a, \varepsilon), \varepsilon) d s
$$


satisfies $\dot{Z}=A(t) \cdot Z+\varepsilon \cdot[q(t, y, \varepsilon)-Q q(t, y, \varepsilon)]$ and also $P Z=0$. Thus we get $P w(t)=P a^{*}(t)+P Z(t)=a^{*}(t)$.

Without difficulty one can show that $\mathscr{F}$ is a contraction map in $S_{0}$ for $0 \leqq|\varepsilon| \leqq \varepsilon_{1}$ and $\varepsilon_{1}$ sufficiently small. Thus $\mathscr{F}$ has a unique fixed point $y^{*}(t)=y^{*}(t, a, \varepsilon)$ given by

$$
y^{*}(t)=a^{*}(t)+\varepsilon \cdot \int_{0}^{T} G(t, s) \cdot q\left(s, y^{*}(s, a, \varepsilon), \varepsilon\right) d s .
$$

Again from (2.6) and (2.9) we see that $y^{*}(t)$ is a solution of (3.1). The contraction mapping principle states that the solution $y^{*}(t)$ can be obtained as the limit of a sequence $\left\{y^{k}(t)\right\}$ defined by

$$
\begin{gathered}
y_{0}(t)=a^{*}(t)=\Phi(t) \cdot a \\
y^{k+1}(t)=\mathscr{F} y^{k}(t)=a^{*}(t)+\varepsilon \cdot \int_{0}^{T} G(t, s) \cdot q\left(s, y^{k}(s), \varepsilon\right) d s .
\end{gathered}
$$

From (3.4) we conclude $y(t, a, 0)=a^{*}(t)$. To prove uniqueness, let $y_{1}=y_{1}(t, \varepsilon)$ be another solution of $(3.1)$ in $S_{0}$ for $|\varepsilon| \leqq \varepsilon_{1}$. Let us define $w(t)=y_{1}(t)-a^{*}(t)$. Then $w$ is a $T$-periodic solution of

$$
\dot{w}=A(t) \cdot w+\varepsilon \cdot\left[q\left(t, y_{1}, \varepsilon\right)-Q q\left(t, y_{1}, \varepsilon\right)\right]
$$

satisfying $P w=0$. It follows now from (2.6) and Lemma 2 that $y_{1}$ is a fixed point of $\mathscr{F}$, which can only mean that $y_{1}=y^{*}$.

REMARK. Consider the special case when $A(t)$ is a constant matrix of the form diag $(0, B)$, where $B$ is an $(n-k) \times(n-k)$ matrix such that $\dot{X}=B \cdot X$ has no $T$-periodic solution $X(t)$. Thus (1.2) has precisely $k$ linearly independent constant solutions, and so we may choose an orthonormal basis of $S_{1}$ to be the first $k$ unit $n$-vectors in $R^{n}$. Then $\Phi(t)$ is the $n \times k$ matrix whose only nonzero terms are $k 1$ 's along the diagonal. Clearly we may choose the same basis for $S_{2}$, and so $\Psi(t)=$ $\Phi(t)$. Thus $P f=Q f$ for all $f \in S$ and the spaces $S_{1}$ and $S_{2}$ coincide. This is the case treated in Chapter 6 of [5].

THEOREM 2. Let $y^{*}(t)=y^{*}(t, a, \varepsilon)$ be the solution of (3.1) defined for all $a \in R^{k}$ and $\varepsilon$ such that $0 \leqq|\varepsilon| \leqq \varepsilon_{1}$ and $V\left(a^{*}\right)=V(\Phi(t) \cdot a) \leqq b$. If there exists an $\varepsilon_{2} \leqq \varepsilon_{1}$ and a continuous function $a=a(\varepsilon)$ for $|\varepsilon| \leqq \varepsilon_{2}$ such that

$$
Q q\left(t, y^{*}(t, a(\varepsilon), \varepsilon), \varepsilon\right)=0
$$

then $y^{*}(t, a(\varepsilon), \varepsilon)$ is a T-periodic solution of (1.1) for $|\varepsilon| \leqq \varepsilon_{2}$. Conversely, suppose (1.1) has a T-periodic solution $\bar{y}=\bar{y}(t, \varepsilon)$ for $|\varepsilon| \leqq$ $\varepsilon_{2} \leqq \varepsilon_{1}$, continuous in $\varepsilon$ with $V(P \bar{y})<R$. Then for some continuous function $a(\varepsilon)$ satisfying $(3.5)$ we have $\bar{y}(t, \varepsilon)=y^{*}(t, a(\varepsilon), \varepsilon)$ for $|\varepsilon| \leqq \varepsilon_{2}$ 
In this sense we say that the existence of a continuous function $a(\varepsilon)$ satisfying (3.5) is a necessary and sufficient condition for the existence of a periodic solution of (1.1).

Proof. The sufficiency of the condition is obvious. Conversely, given $\bar{y}=\bar{y}(t, \varepsilon)$ define $a(\varepsilon)^{\prime}=\left(\left(\bar{y}, \varphi_{1}\right),\left(\bar{y}, \varphi_{2}\right), \cdots,\left(\bar{y}, \varphi_{k}\right)\right)$. Then for $a^{*}(t, \varepsilon)=\Phi(t) \cdot a(\varepsilon)$ we have $P \bar{y}(t, \varepsilon)=a^{*}(t, \varepsilon)$. Clearly $a(\varepsilon)$ is continuous and by assumption $V(P \bar{y})=V P(\bar{y}(\cdot, \varepsilon))=V\left(a^{*}(\cdot, \varepsilon)\right)<R$. Thus for some $b, d$ with $0<b<d<R$ we have $V\left(a^{*}(\cdot, \varepsilon)\right) \leqq b<R$ for $0 \leqq$ $|\varepsilon| \leqq \varepsilon_{2}$. Since $\bar{y}$ is a solution of (1.1) we obviously have

$$
\begin{aligned}
(I-Q)[\dot{\bar{y}}-A(t) \cdot \bar{y}-\varepsilon \cdot q(t, \bar{y}, \varepsilon)] & =0 \\
Q[\dot{\bar{y}}-A(t) \cdot \bar{y}-\varepsilon \cdot q(t, \bar{y}, \varepsilon)] & =0 .
\end{aligned}
$$

Since $\bar{y}$ is assumed known we can regard it as a solution of an equation of the form (1.3). From Lemma 1 this implies $Q q(t, \bar{y}(t, \varepsilon), \varepsilon)=0$. Thus the above equations reduce to

$$
\begin{gathered}
\dot{\bar{y}}=A(t) \cdot \bar{y}+\varepsilon \cdot[q(t, \bar{y}, \varepsilon)-Q q(t, \bar{y}, \varepsilon)] \\
Q q(t, \bar{y}, \varepsilon)=0
\end{gathered}
$$

for $0 \leqq|\varepsilon| \leqq \varepsilon_{2}$. From Theorem 1 , however, the function $y^{*}(t, a(\varepsilon), \varepsilon)$ is the only $T$-periodic solution of (3.6) satisfying

$$
P\left(y^{*}(t, a(\varepsilon), \varepsilon)\right)=a^{*}(t, \varepsilon)
$$

for $|\varepsilon| \leqq \varepsilon_{1}$. Since $\varepsilon_{2} \leqq \varepsilon_{1}$, from (3.6) we conclude $\bar{y}(t, \varepsilon)=y^{*}(t, a(\varepsilon), \varepsilon)$ for $|\varepsilon| \leqq \varepsilon_{2}$ and from (3.7) we see that $a(\varepsilon)$ satisfies (3.5).

REMark. Condition (3.5) is most easily verified, of course, when the implicit function theorem can be applied in the manner of Chapter 6 of [5]. If this fails, then one can use (3.4) to find approximate determining equations for (1.1) involving the successive approximations $y_{0}, y^{1}, \cdots y^{k}$, as done in Chapter 7 of [5]. One advantage of the present methods is that these higher order equations can be explicitly written out, due to the constructive method used to define the kernel $G(t, s)$ of the integral operator $\mathscr{F}$.

\section{BIBLIOGRAPHY}

1. H. Antosiewicz, Boundary value problems for nonlinear ordinary differential equations, Pacific J. Math. 15 (1966), 191-197.

2. S. Bancroft, J. K. Hale and D. Sweet, Alterenative problems for nonlinear functional equations, J. Differential Eqs. 4 (1968), 40-56.

3. F. A. Ficken, Linear transformations and matrices, Prentice Hall, Englewood Cliffs, N. J., 1967. 
4. K. O. Friedrichs, Functional analysis and applications, Inst. Math. Sci., New York Univ., New York, 1956.

5. J. K. Hale, Oscillations in nonlinear systems, McGraw-Hill, New York, 1963.

6. D. C. Lewis, On the role of first integrals in the perturbation of periodic solutions, Ann. of Math. 63 (1956), 535-548.

7. W. S. Loud, Generalized inverses and generalized Green's functions, SIAM J. Appl. Math. 14 (1966), 342-369.

8. W. T. Reid, Generalized Green's matrices for two-point boundary problems. SIAM J. Appl. Math. 15 (1967), 856-870.

Received June 24, 1968. The author is indebted to Professor Arnold Stokes of Georgetown University for his encouragement and advice during the work leading to this paper.

Mobil Research and Development Corporation

PRINCETON, NEW JERSEY 


\section{PACIFIC JOURNAL OF MATHEMATICS}

\section{EDITORS}

H. ROYDEN
Stanford University
Stanford, California

\section{R. R. PHELPS}

University of Washington

Seattle, Washington 98105
J. DUGUNDJI

Department of Mathematics

University of Southern California

Los Angeles, California 90007

\section{RICHARD ARENS}

University of California

Los Angeles, California 90024

\section{ASSOCIATE EDITORS}

E. F. BeCKenbaCh
B. H. NEUMANN

F. WOLF
K. YoshidA

\section{SUPPORTING INSTITUTIONS}

\author{
UNIVERSITY OF BRITISH COLUMBIA \\ CALIFORNIA INSTITUTE OF TECHNOLOGY \\ UNIVERSITY OF CALIFORNIA \\ MONTANA STATE UNIVERSITY \\ UNIVERSITY OF NEVADA \\ NEW MEXICO STATE UNIVERSITY \\ OREGON STATE UNIVERSITY \\ UNIVERSITY OF OREGON \\ OSAKA UNIVERSITY \\ UNIVERSITY OF SOUTHERN CALIFORNIA
}

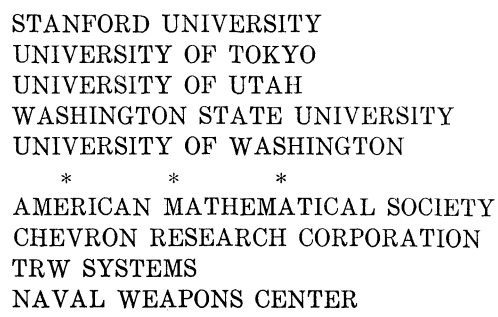

The Supporting Institutions listed above contribute to the cost of publication of this Journal, but they are not owners or publishers and have no responsibility for its content or policies.

Mathematical papers intended for publication in the Pacific Journal of Mathematics should be in typed form or offset-reproduced, double spaced with large margins. Underline Greek letters in red, German in green, and script in blue. The first paragraph or two must be capable of being used separately as a synopsis of the entire paper. It should not contain references to the bibliography. Manuscripts, in duplicate if possible, may be sent to any one of the four editors. Please classify according to the scheme of Math. Rev. 36, 1539-1546. All other communications to the editors should be addressed to the managing editor, Richard Arens, University of California, Los Angeles, California, 90024.

50 reprints are provided free for each article; additional copies may be obtained at cost in multiples of 50 .

The Pacific Journal of Mathematics is published monthly. Effective with Volume 16 the price per volume (3 numbers) is $\$ 8.00$; single issues, $\$ 3.00$. Special price for current issues to individual faculty members of supporting institutions and to individual members of the American Mathematical Society: $\$ 4.00$ per volume; single issues $\$ 1.50$. Back numbers are available.

Subscriptions, orders for back numbers, and changes of address should be sent to Pacific Journal of Mathematics, 103 Highland Boulevard, Berkeley, California, 94708.

PUBLISHED BY PACIFIC JOURNAL OF MATHEMATICS, A NON-PROFIT CORPORATION

Printed at Kokusai Bunken Insatsusha (International Academic Printing Co., Ltd.), 7-17, Fujimi 2-chome, Chiyoda-ku, Tokyo, Japan. 


\section{Pacific Journal of Mathematics \\ Vol. 29, No. $2 \quad$ June, 1969}

Bruce Langworthy Chalmers, On boundary behavior of the Bergman kernel function and related domain functionals ................... 243

William Eugene Coppage, Peirce decomposition in simple Lie-admissible power-associative rings .............................. 251

Edwin Duda, Compactness of mappings...................... 259

Earl F. Ecklund Jr., On prime divisors of the binomial coefficient......... 267

Don E. Edmondson, A modular topological lattice ............... 271

Phillip Alan Griffith, A note on a theorem of Hill ................... 279

Marcel Herzog, On finite groups with independent cyclic Sylow

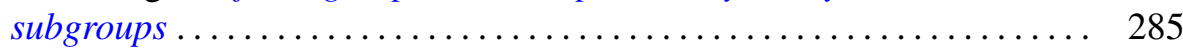

James A. Huckaba, Extensions of pseudo-valuations................. 295

S. A. Huq, Semivarieties and subfunctors of the identity functor ........ 303

I. Martin (Irving) Isaacs and Donald Steven Passman, Finite groups with small character degrees and large prime divisors. II ............ 311

Carl Kallina, A Green's function approach to perturbations of periodic

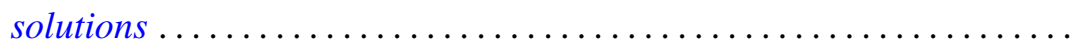

$\mathrm{Al}$ (Allen Frederick) Kelley, Jr., Analytic two-dimensional subcenter manifolds for systems with an integral ....................

Alistair H. Lachlan, Initial segments of one-one degrees ............ 351

Marion-Josephine Lim, Rank k Grassmann products ............. 367

Raymond J. McGivney and William Henry Ruckle, Multiplier algebras of

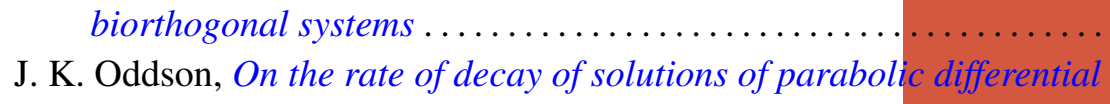

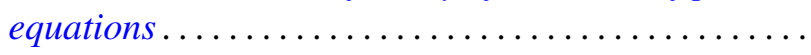

Helmut R. Salzmann, Geometries on surfaces ........... .

Annemarie Schlette, Artinian, almost abelian groups and their groups of automorphisms ............................

Edgar Lee Stout, Additional results on modules over polydisc algebras ...

Lajos Tamássy, A characteristic property of the sphere . .

Mark Lawrence Teply, Some aspects of Goldie's torsion theory. ...

Freddie Eugene Tidmore, Extremal structure of star-shaped sets ...

461

Leon Jarome Weill, Unconditional and shrinking bases in locally convex spaces... 